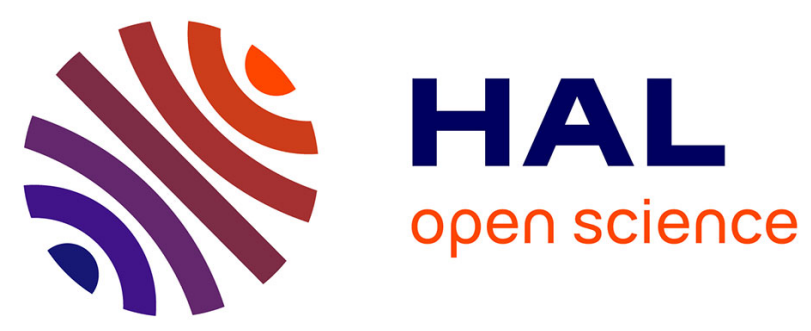

\title{
Diffusion on a random lattice: Weak-disorder expansion in arbitrary dimension
}

\author{
Bernard Derrida, J. Luck
}

\section{To cite this version:}

Bernard Derrida, J. Luck. Diffusion on a random lattice: Weak-disorder expansion in arbitrary dimension. Physical Review B, 1983, 28 (12), pp.7183-7190. 10.1103/PhysRevB.28.7183 . hal03285932

\section{HAL Id: hal-03285932 \\ https://hal.science/hal-03285932}

Submitted on 19 Jul 2021

HAL is a multi-disciplinary open access archive for the deposit and dissemination of scientific research documents, whether they are published or not. The documents may come from teaching and research institutions in France or abroad, or from public or private research centers.
L'archive ouverte pluridisciplinaire HAL, est destinée au dépôt et à la diffusion de documents scientifiques de niveau recherche, publiés ou non, émanant des établissements d'enseignement et de recherche français ou étrangers, des laboratoires publics ou privés. 


\title{
Diffusion on a random lattice: Weak-disorder expansion in arbitrary dimension
}

\author{
B. Derrida and J. M. Luck \\ Département de Physique Générale, Institut de Recherche Fondamentale, Service de Physique Théorique, \\ Commissariat à l'Energie Atomique, Centre d'Etudes Nucléaires de Saclay, \\ F-91191 Gif-sur-Yvette Cedex, France
}

(Received 2 May 1983)

\begin{abstract}
We consider a random-hopping model on a regular lattice. We describe a method of calculating the drift velocity, the diffusion tensor, and the conductivity. The method works for symmetric as well as for nonsymmetric hopping rates. We can use this way to obtain systematic weak-disorder expansions. In the symmetric case we compare the results of the expansion with those of the effective-medium approximation. In the nonsymmetric case, the expansion shows that the upper critical dimension is 2 . At $d=2$, we conjecture logarithmic corrections in the velocity.
\end{abstract}

\section{INTRODUCTION}

Classical diffusion in a random medium has been a subject of growing interest in the last few years. There have been considerable efforts to explain the conductivity properties $^{1,2}$ of one-dimensional disordered conductors, and now the problem can be solved by various methods ${ }^{3}$ : the effective-medium approximation, ${ }^{4-6}$ the renormalization group, ${ }^{7}$ the replica trick, ${ }^{8}$ etc. Another subject of great interest is the problem of diffusion on percolation clusters $^{9-11}$ in the neighborhood of the threshold. Several approaches were developed: Monte Carlo simulations, ${ }^{12}$ effective-medium approximation, ${ }^{4,6,13}$ random walks on fractals, ${ }^{14}$ most of the recent works on this subject being motivated by a conjecture of Alexander and Orbach. ${ }^{15}$ In these two problems, the hopping rates were always assumed to be symmetric, i.e., the probability $W_{y, x}$ of jumping from site $x$ to site $y$ was equal to $W_{x, y}$. The nonsymmetric case, although it has been less studied by physicists, is also very interesting for several reasons.

(i) Firstly, it is a very important problem in probability $^{16-18}$ theory, and several nice results have been obtained in one dimension.

(ii) Secondly, it is one of the easiest disordered systems to study: It has the advantage of being the generalization to the disordered case of the random-walk problem, which is one of the best understood problems of statistical mechanics. However, the effect of disorder, even when the distribution of hopping rates is very narrow, has nontrivial effects: One finds, for example, in one-dimension exponents varying continuously with the distribution of hopping rates. ${ }^{16,17,19,20}$ One finds also a typical effect of some disordered systems, namely that different quantities such as the velocity and the diffusion constant are singular at different points. ${ }^{21}$ Therefore, there is no doubt that a better understanding of the effect of disorder in this random-hopping problem will be useful in the study of more complicated disordered systems.

(iii) A third interest for nonsymmetric hopping problems is that in physical systems one can consider the effect of an external electric field ${ }^{22,23}$ or of local electric random fields. Both effects give rise to nonsymmetric hopping rates, the ratio between $W_{x, y}$ and $W_{y, x}$ being related to the value of the electric field between sites $x$ and $y$.

(iv) Lastly, it has been recently pointed out that the one-dimensional case could explain the famous problem of $1 / f$ noise. $^{24}$

In this paper we present a weak disorder expansion of the velocity, the diffusion tensor, and the conductivity of the nonsymmetric hopping problem. Since it is known in one dimension ${ }^{19-24}$ that new features appear even for narrow distributions of hopping rates, it is reasonable to think that several interesting properties can be extracted from these expansions in higher dimension. The method used to generate these expansions is a generalization of a calculation done recently for a one-dimensional case. ${ }^{22}$ In principle these expansions can be carried out to any order in the width of the distribution of hopping rates. Then, to illustrate the interest of these expansions we analyze two situations: the symmetric case, where we compare the results with those of the effective-medium theory and the more general nonsymmetric case. In that case we find that $d=2$ is the upper critical dimensionality because the weak disorder expansion is singular for small velocities when $d \leq 2$.

Our starting point is a master equation

$$
\frac{d P_{x}}{d t}=\sum_{x^{\prime}}\left(W_{x, x^{\prime}} P_{x^{\prime}}-W_{x^{\prime}, x} P_{x}\right),
$$

which describes the time evolution of $P_{x}(t)$, the probability for one particle to be on site $x$ at time $t$. By definition $x$ is a point of a $d$-dimensional lattice

$$
x=\overrightarrow{\mathrm{x}}=\left(x_{1}, x_{2}, \ldots, x_{\mu}, \ldots, x_{d}\right)
$$

whereas $W_{x^{\prime}, x} d t$ is the probability of jumping from site $x$ to site $x^{\prime}$ during $d t$. In this paper we shall consider that the $W_{x, x^{\prime}}$ are randomly distributed according to a distribution which depends only on the distance $x^{\prime}-x$. We shall limit ourselves to the case where the $W_{x, x^{\prime}}$ are independent random variables except that the two hopping rates $W_{x^{\prime}, x}$ and $W_{x, x^{\prime}}$ lying on the same bond will be possibly correlat- 
ed. This restriction of considering independent $W_{x, x^{\prime}}$ is not absolutely necessary but it will simplify our final expressions. We shall write

$$
W_{x, x^{\prime}}=a\left(x-x^{\prime}\right)+\delta W_{x, x^{\prime}}
$$

and calculate the quantities of interest up to a given power of the $\delta W_{x, x^{\prime}}$. The zeroth order corresponds to a random walk on a pure lattice with hopping rates $a\left(x-x^{\prime}\right)$. Notice that we do not need to restrict the jumps to nearest neighbors. We shall express our results as functions of the successive moments $c_{n p}\left(x-x^{\prime}\right)$ of the $\delta W_{x, x^{\prime}}$ defined by

$$
c_{n p}\left(x-x^{\prime}\right)=\left\langle\left(\delta W_{x, x^{\prime}}\right)^{n-p}\left(\delta W_{x^{\prime}, x}\right)^{p}\right\rangle .
$$

The paper is organized as follows. In Secs. II and III we write the equations which lead to the expressions of the velocity, the diffusion tensor, and the conductivity. In Sec. IV we expand the solutions of these equations in powers of the $\delta W_{x, x^{\prime}}$ and we obtain the first corrections due to the presence of disorder. In Sec. $V$ we give two applications of these expansions. We compare the results of this expansion with the prediction of the effective-medium theory in the symmetric case. Then we show that the weak disorder expansion becomes singular in the nonsymmetric case for $d \leq 2$ and we examine the consequences on the critical behaviors of the velocity.

\section{THE VELOCITY AND THE DIFFUSION TENSOR}

In this section we derive the expressions which give the velocity and the diffusion tensor. The method ${ }^{21}$ we use to obtain these quantities consists of describing the properties of the steady state of a periodic $d$-dimensional hypercubic lattice whose elementary cell contains $\Lambda=\lambda^{d}$ sites. The hopping rates in the elementary cell are arbitrary. The reason why we need to make the calculations on a periodic lattice is that the steady state will be characterized, in particular, by the probabilities $P_{x}$ of being on sites $x$ in the long-time limit. For an infinite lattice, the $P_{x}$ vanish and the idea of a steady state is not valid. However, if the lattice is periodic, the sum of $P_{x}$ over all the points which can be deduced from $x$ by the translations of the lattice has a finite limit.

Because the problem is periodic with a period $\lambda$ in each direction we have

$$
W_{x, x^{\prime}}=W_{x+n \lambda, x^{\prime}+n \lambda},
$$

where $n$ is a $d$-dimensional vector with integer components

$$
n=\left(n_{1}, n_{2}, \ldots, n_{\mu}, \ldots, n_{d}\right) \text {. }
$$

It is convenient to introduce two quantities

$$
\begin{aligned}
\widetilde{Q}_{x}(t) & =\sum_{n} P_{x+n \lambda}(t), \\
\widetilde{\mathrm{S}}_{x}(t) & =\sum_{n}(\overrightarrow{\mathrm{x}}+\overrightarrow{\mathrm{n}} \lambda) P_{x+n \lambda}(t),
\end{aligned}
$$

where the sum runs over all vectors $n \in Z^{d}$ The $\widetilde{\overrightarrow{\mathrm{S}}}_{x}(t)$ are $d$-dimensional vectors as $x$ and $n$.
It is easy to obtain the time evolution of $\widetilde{Q}_{x}(t)$ and $\widetilde{\vec{S}}_{x}(t)$ from the master equation (1):

$$
\begin{aligned}
& \frac{d \widetilde{Q}_{x}}{d t}=\sum_{x^{\prime}}\left(W_{x, x^{\prime}} \widetilde{Q}_{x^{\prime}}-W_{x^{\prime}, x} \widetilde{Q}_{x}\right) \\
& \frac{\widetilde{\overrightarrow{\mathrm{S}}}}{d t}=\sum_{x^{\prime}}\left(W_{x, x^{\prime}} \widetilde{\overrightarrow{\mathrm{S}}}_{x^{\prime}}-W_{x^{\prime}, x} \widetilde{\overrightarrow{\mathrm{S}}}_{x}\right)+\sum_{x^{\prime}}\left(\overrightarrow{\mathrm{x}}-\overrightarrow{\mathrm{x}}^{\prime}\right) W_{x, x^{\prime}} \widetilde{Q}_{x}
\end{aligned}
$$

As in the one-dimensional case, ${ }^{21}$ we expect that in the long-time limit $(t \rightarrow \infty)$, these quantities have the following behaviors:

$$
\begin{aligned}
& \widetilde{Q}_{x}(t) \rightarrow Q_{x} \text { if } t \rightarrow \infty, \\
& \widetilde{\overrightarrow{\mathrm{S}}}_{x}(t) \rightarrow \overrightarrow{\mathrm{S}}_{x} t+\overrightarrow{\mathrm{T}}_{x} \text { if } t \rightarrow \infty .
\end{aligned}
$$

If we replace $\widetilde{Q}_{x}$ and $\widetilde{\vec{S}}_{x}$ by their long-time expansions in Eqs. (9) and (10), we find that $Q_{x}, \overrightarrow{\mathrm{S}}_{x}$, and $\overrightarrow{\mathrm{T}}_{x}$ must verify

$$
\begin{aligned}
& \sum_{x^{\prime}}\left(W_{x, x^{\prime}} Q_{x^{\prime}}-W_{x^{\prime}, x} Q_{x}\right)=0, \\
& \sum_{x^{\prime}}\left(W_{x, x^{\prime}} \overrightarrow{\mathrm{S}}_{x^{\prime}}-W_{x^{\prime}, x} \overrightarrow{\mathrm{S}}_{x}\right)=0, \\
& \sum_{x^{\prime}}\left(W_{x, x^{\prime}} \overrightarrow{\mathrm{T}}_{x^{\prime}}-W_{x^{\prime}, x} \overrightarrow{\mathrm{T}}_{x}\right)+\sum_{x^{\prime}}\left(\overrightarrow{\mathrm{x}}-\overrightarrow{\mathrm{x}}^{\prime}\right) W_{x, x^{\prime}} Q_{x}=\overrightarrow{\mathrm{S}}_{x} .
\end{aligned}
$$

We are now going to show that if we know the solutions of (13)-(15), we can calculate the velocity and the diffusion tensor.

If we define $\left\langle x_{\mu}(t)\right\rangle$ and $\left\langle x_{\mu}(t) x_{v}(t)\right\rangle$ by

$$
\begin{aligned}
& \left\langle x_{\mu}(t)\right\rangle=\sum_{x} x_{\mu} P_{x}(t), \\
& \left\langle x_{\mu} x_{v}(t)\right\rangle=\sum_{x} x_{\mu} x_{v} P_{x}(t)
\end{aligned}
$$

(in this section, the angular brackets denote the average over the random walk), we can calculate the components of the velocity $\vec{v}$ and of the diffusion tensor $D$ by

$$
\begin{aligned}
& v_{\mu}=\lim _{t \rightarrow \infty} \frac{d}{d t}\left\langle x_{\mu}\right\rangle, \\
& D_{\mu \nu}=\frac{1}{2} \lim _{t \rightarrow \infty} \frac{d}{d t}\left(\left\langle x_{\mu} x_{\nu}\right\rangle-\left\langle x_{\mu}\right\rangle\left\langle x_{v}\right\rangle\right) .
\end{aligned}
$$

Let us first calculate the velocity

$$
\begin{aligned}
\frac{d}{d t}\left\langle x_{\mu}\right\rangle & =\sum_{x} x_{\mu} \frac{d P_{x}}{d t} \\
& =\sum_{x, x^{\prime}} x_{\mu}\left(W_{x, x^{\prime}} P_{x^{\prime}}-W_{x^{\prime}, x} P_{x}\right) \\
& =\sum_{x, x^{\prime}}\left(x_{\mu}^{\prime}-x_{\mu}\right) W_{x^{\prime}, x} P_{x}
\end{aligned}
$$


In the limit $t \rightarrow \infty$, one obtains

$$
v_{\mu}=\sum_{x \in \Lambda} \sum_{x^{\prime}}\left(x_{\mu}^{\prime}-x_{\mu}\right) W_{x^{\prime}, x} Q_{x}
$$

where the $Q_{x}$ are the normalized solutions of Eq. (13):

$$
\sum_{x \in \Lambda} Q_{x}=1
$$

The calculation of the diffusion tensor is similar to the calculation of the velocity. One finds in the long-time limit

$$
\begin{aligned}
\frac{d}{d t}\left\langle x_{\mu} x_{v}\right\rangle \simeq & t \sum_{x \in \Lambda} \sum_{x^{\prime}}\left[\left(x_{\mu}^{\prime}-x_{\mu}\right) W_{x^{\prime}, x}\left(S_{x}\right)_{v}+\left(x_{v}^{\prime}-x_{v}\right) W_{x^{\prime}, x}\left(S_{x}\right)_{\mu}\right] \\
& +\sum_{x \in \Lambda} \sum_{x^{\prime}}\left[\left(x_{\mu}^{\prime}-x_{\mu}\right) W_{x^{\prime}, x}\left(T_{x}\right)_{v}+\left(x_{v}^{\prime}-x_{v}\right) W_{x^{\prime}, x}\left(T_{x}\right)_{\mu}\right]+\sum_{x \in \Lambda} \sum_{x^{\prime}}\left(x_{\mu}^{\prime}-x_{\mu}\right)\left(x_{v}^{\prime}-x_{v}\right) W_{x^{\prime}, x} Q_{x}, \\
\frac{d}{d t}\left\langle x_{\mu}\right\rangle\left\langle x_{v}\right\rangle & =2 t \sum_{x \in \Lambda} \sum_{y \in \Lambda}\left(S_{x}\right)_{\mu}\left(S_{y}\right)_{v}+\sum_{x \in \Lambda} \sum_{y \in \Lambda}\left[\left(T_{x}\right)_{\mu}\left(S_{y}\right)_{v}+\left(S_{x}\right)_{\mu}\left(T_{y}\right)_{v}\right] .
\end{aligned}
$$

The solution $Q_{x}$ of Eq. (13) is unique provided that one cannot divide the sites into two subsets of sites $x$ between which the $W_{x x^{\prime}}$ would be zero. If we assume that this is not the case, there is only one normalized solution of (13). Therefore the solution of (14) is

$$
\overrightarrow{\mathbf{S}_{x}}=\overrightarrow{\mathrm{V}} Q_{x}
$$

where $\vec{V}$ is $a$ priori an arbitrary constant vector. It is very easy to find that

$$
\vec{v}=\vec{V}
$$

by summing Eq. (15) over $x$ and by comparing the left-hand side to Eq. (21). Then when one sets relations (23) and (24) into (19), one obtains

$$
\begin{aligned}
D_{\mu \nu}= & \frac{1}{2} \sum_{x \in \Lambda} \sum_{x^{\prime}}\left\{\left[\left(x_{\mu}^{\prime}-x_{\mu}\right)\left(T_{x}\right)_{v}+\left(x_{v}^{\prime}-x_{v}\right)\left(T_{x}\right)_{\mu}\right] W_{x^{\prime}, x}+\left[\left(x_{\mu}^{\prime}-x_{\mu}\right)\left(x_{v}^{\prime}-x_{v}\right) Q_{x} W_{x^{\prime}, x}\right]\right\} \\
& -\frac{1}{2} V_{\mu} \sum_{x \in \Lambda}\left(T_{x}\right)_{v}-\frac{1}{2} V_{v} \sum_{x \in \Lambda}\left(T_{x}\right)_{\mu} .
\end{aligned}
$$

One should notice that because of (25) and (26), the term linear in time in (23) is canceled by the term linear in time in (24). In concluding this section we have shown that if we can solve Eqs. (13)-(15), then we know how to calculate the velocity and diffusion tensor [Eqs. (21) and (27)].

\section{THE FREQUENCY-DEPENDENT CONDUCTIVITY}

We are now going to obtain a general expression of the ac conductivity. The method that we follow here is a generalization to arbitrary dimension of a formulation yet used in one dimension. ${ }^{22}$ There is a slight modification in the definition which has the effect of adding to the conductivity a constant term, independent of frequency, and therefore the physical meaning of the results remains unchanged.

As in Sec. II, we work here on a finite lattice of $\Lambda$ sites with periodic boundary conditions. We consider that the effect of a time-dependent uniform electric field $\overrightarrow{\mathrm{E}} e^{i \omega t}$ of frequency $\omega$ is to give a time dependence to the hopping rates $W_{x, x^{\prime}}$. In the presence of the field the $W_{x, x^{\prime}}$ should be replaced by $\widetilde{W}_{x, x^{\prime}}(t)$,

$$
\widetilde{W}_{x, x^{\prime}}(t)=W_{x, x^{\prime}} \exp \left[\overrightarrow{\mathrm{E}} \cdot\left(\overrightarrow{\mathrm{x}}-\overrightarrow{\mathrm{x}}^{\prime}\right) e^{i \omega t}\right]
$$

linearize this relation

$$
\widetilde{W}_{x, x^{\prime}}(t)=W_{x, x^{\prime}}\left[1+\overrightarrow{\mathrm{E}} \cdot\left(\overrightarrow{\mathrm{x}}-\overrightarrow{\mathrm{x}}^{\prime}\right) e^{i \omega t}\right] .
$$

We have now to study the long-time behavior of the master equation with the hopping rates $\widetilde{W}_{x, x^{\prime}}$,

$$
\frac{d P_{x}}{d t}=\sum_{x^{\prime}}\left(\widetilde{W}_{x, x^{\prime}} P_{x^{\prime}}-\widetilde{W}_{x^{\prime}, x} P_{x}\right)
$$

In the long-time limit, the $P_{x}$ have the following time dependence:

$$
P_{x}(t)=Q_{x}+\overrightarrow{\mathrm{R}}_{x} \cdot \overrightarrow{\mathrm{E}} e^{i \omega t} .
$$

If we put (31) into (30), we find that the zeroth order and the first order in the field give the equations verified by $Q_{x}$ and $\overrightarrow{\mathbf{R}}_{x}$ :

$$
\sum_{x^{\prime}}\left(W_{x, x^{\prime}} Q_{x^{\prime}}-W_{x^{\prime}, x} Q_{x}\right)=0
$$

and

$$
\begin{aligned}
i \omega \overrightarrow{\mathbf{R}}_{\boldsymbol{x}}= & \sum_{x^{\prime}}\left(W_{x, x^{\prime}} \overrightarrow{\mathbf{R}}_{x^{\prime}}-W_{x^{\prime}, x} \overrightarrow{\mathrm{R}}_{x}\right) \\
& +\sum_{x^{\prime}}\left[W_{x, x^{\prime}}\left(\overrightarrow{\mathrm{x}}-\overrightarrow{\mathrm{x}}^{\prime}\right) Q_{x^{\prime}}-W_{x^{\prime}, x}\left(\overrightarrow{\mathrm{x}}^{\prime}-\overrightarrow{\mathrm{x}}\right) Q_{x}\right]
\end{aligned}
$$

Since the conductivity describes only linear effects we can 
One should notice that if one sums this equation over $x$, one finds

$$
\sum_{x} \vec{R}_{x}=\overrightarrow{0}
$$

which expresses the conservation of the probability.

The velocity at time $t$ is always given by

$$
\begin{aligned}
\overrightarrow{\mathrm{v}}(t)=\sum_{x} \overrightarrow{\mathrm{x}} \frac{d P_{x}}{d t} & =\sum_{x} \sum_{x^{\prime}} \overrightarrow{\mathrm{x}}\left(\widetilde{W}_{x, x^{\prime}} P_{x^{\prime}}-\widetilde{W}_{x^{\prime}, x} P_{x}\right) \\
& =\sum_{x} \sum_{x^{\prime}}\left(\overrightarrow{\mathrm{x}}^{\prime}-\overrightarrow{\mathrm{x}}\right) \widetilde{W}_{x^{\prime}, x} P_{x} .
\end{aligned}
$$

If we replace now the $P_{x}$ and the $\widetilde{W}_{x, x^{\prime}}$ by their expressions (29) and (31) in the long-time limit, one finds that the component $v_{\mu}(t)$ of the velocity has the following form:

$$
v_{\mu}(t)=V_{\mu}+\sum_{\nu} \sigma_{\mu v} E_{v} e^{i \omega t},
$$

where $V$ is the drift velocity that we have obtained in Sec. II,

$$
V_{\mu}=\sum_{x \in \Lambda} \sum_{x^{\prime}}\left(x_{\mu}^{\prime}-x_{\mu}\right) W_{x^{\prime}, x} Q_{x},
$$

and where the tensor $\sigma_{\mu \nu}$ is the conductivity tensor

$$
\begin{array}{r}
\sigma_{\mu v}=\sum_{x \in \Lambda}\left(\sum_{x^{\prime}}\left(x_{\mu}^{\prime}-x_{\mu}\right) W_{x^{\prime}, x}\left(x_{v}^{\prime}-x_{v}\right) Q_{x}\right. \\
\left.+\sum_{x^{\prime}}\left(x_{\mu}^{\prime}-x_{\mu}\right) W_{x^{\prime}, x}\left(R_{x}\right)_{v}\right) .
\end{array}
$$

So we see that to calculate the conductivity tensor we need to find the $Q_{x}$ and $\overrightarrow{\mathrm{R}}_{x}$ which solve Eqs. (13) and (32), and then we can use formula (35).

\section{THE WEAK DISORDER EXPANSION}

We saw in Secs. II and III that one can calculate the velocity, the diffusion tensor, and the ac conductivity by using Eqs. (21), (27), and (35), which require the solutions of (13), (15), and (32). It is almost always impossible to solve these equations except in the pure case or in some one-dimensional situations. However, it is easy to expand the solutions of these equations in increasing powers of the $\delta W_{x, x^{\prime}}$. Let us show here how this expansion works to find the solutions of (15) and (32).

If we replace the $W_{x, x^{\prime}}$ by their expression (3), one can write the solutions $Q_{x}$ of (13) in the following form:

$$
Q_{x}=\left(1+\varphi_{x}\right) / \Lambda,
$$

where the $\varphi_{x}$ represents the change in the solutions of (13) due to the $\delta W_{x, x^{\prime}}$. The $\varphi_{x}$ must obey

$$
\begin{aligned}
\sum_{x^{\prime}}\left[a\left(x^{\prime}-x\right)\right. & \left.\varphi_{x}-a\left(x-x^{\prime}\right) \varphi_{x^{\prime}}\right] \\
= & \sum_{x^{\prime}}\left[\delta W_{x, x^{\prime}}\left(1+\varphi_{x^{\prime}}\right)-\delta W_{x^{\prime}, x}\left(1+\varphi_{x}\right)\right]
\end{aligned}
$$

Equation (37) is written in such a way that the $\varphi_{x}$ can be found iteratively,

$$
\varphi_{x}=\sum_{y, y^{\prime} \in \Lambda} G_{\Lambda}\left(y^{\prime}-x, y-x\right) \delta W_{y^{\prime}, y}\left(1+\varphi_{y}\right),
$$

where the Green's function $G_{\Lambda}$ is solution of

$$
\begin{gathered}
\sum_{x^{\prime}}\left[a\left(x^{\prime}-x\right) G_{\Lambda}\left(y^{\prime}-x, y-x\right)-a\left(x-x^{\prime}\right) G_{\Lambda}\left(y^{\prime}-x^{\prime}, y-x^{\prime}\right)\right] \\
=\sum_{x^{\prime}}\left(\delta_{x, y^{\prime}} \delta_{x^{\prime}, y}-\delta_{x, y} \delta_{x^{\prime}, y^{\prime}}\right)
\end{gathered}
$$

In order to keep the $Q_{x}$ normalized, one must choose the solution $G_{\Lambda}$ of (39) which has the property that for an arbitrary choice of $y$ and $y^{\prime}$

$$
\sum_{x \in \Lambda} G_{\Lambda}\left(y^{\prime}-x, y-x\right)=0
$$

Equation (39) can be solved easily in the Fourier space and one obtains

$$
G_{\Lambda}\left(y^{\prime}, y\right)=\frac{1}{\Lambda} \sum_{q \neq 0}\left[\frac{e^{i q y^{\prime}}-e^{i q y}}{\sum_{z} a(z)\left(1-e^{i q z}\right)}\right]
$$

where the sum runs over all the $q$ of the reciprocal cell except $q=0$, i.e.,

$$
q=\left(\frac{2 \pi n_{1}}{\lambda}, \frac{2 \pi n_{2}}{\lambda}, \ldots, \frac{2 \pi n_{d}}{\lambda}\right)
$$

with $0 \leq n_{i}<\lambda$.

One should notice that the restriction $q \neq 0$ is due to the condition (40). Therefore, we do not need to define (41) for $q=0$ where Eq. (41) is indeterminate.

We can now find the complete expansion of the velocity by replacing $Q_{x}$ by its expansion in Eq. (21). One obtains

$$
\begin{aligned}
V_{\mu}= & \sum_{z} z_{\mu} a(z)+\sum_{z} z_{\mu}\left[\frac{1}{\Lambda} \sum_{x \in \Lambda} \delta W_{x+z, x}\right]+\sum_{z} z_{\mu}\left(\frac{1}{\Lambda} \sum_{x, y, y^{\prime}} \delta W_{x+z, x} G_{\Lambda}\left(y^{\prime}-x, y-x\right) \delta W_{y^{\prime}, y}\right) \\
& +\sum_{z} z_{\mu}\left[\frac{1}{\Lambda} \sum_{x, y, y^{\prime}, t, t^{\prime}} \delta W_{x+z, x} G_{\Lambda}\left(y^{\prime}-x, y-x\right) \delta W_{y^{\prime}, y} G_{\Lambda}\left(t^{\prime}-y, t-y\right) \delta W_{t^{\prime}, t}\right]+\cdots .
\end{aligned}
$$

There is, of course, no difficulty in continuing this expansion to any order in the $\delta W_{y^{\prime}, y}$. In the thermodynamic limit this expansion becomes simpler for two reasons. Firstly, the sums of $\delta W_{x^{\prime}, x}$ can be replaced by the moments $c_{n p}$ defined in Eq. (4). Secondly, the Green's function $G_{\Lambda}$ becomes an integral over the Brillouin zone. For the velocity in the thermo- 
dynamic limit, one gets the following expression:

$$
\begin{aligned}
V_{\mu}= & \sum_{z} z_{\mu} a(z)+\sum_{z} z_{\mu}\left[G(z, 0) c_{2} 0(z)+G(0, z) c_{21}(z)\right] \\
& +\sum_{z} z_{\mu}\left[G^{2}(z, 0) c_{30}(z)+G(z, 0) G(0, z) c_{31}(z)+G(0, z) G(-z, 0) c_{32}(z)+G(0, z) G(0,-z) c_{31}(z)\right]+\cdots
\end{aligned}
$$

and $G\left(y^{\prime}, y\right)$ is just the limit of $G_{\Lambda}\left(y^{\prime}, y\right)$ when $\Lambda \rightarrow \infty$, i.e.,

$$
G\left(y^{\prime}, y\right)=\frac{1}{(2 \pi)^{d}} \int_{0}^{2 \pi} \cdots \int_{0}^{2 \pi} d^{d} q \frac{e^{i q y^{\prime}}-e^{i q y}}{\sum_{z} a(z)\left(1-e^{i q z}\right)}
$$

In cases where the indetermination at $q=0$ would be a problem, one can always use the fact that $G$ is the limit of $G_{\Lambda}$ when $\Lambda \rightarrow \infty$. Although the calculations are slightly longer to obtain the diffusion and the conductivity tensors, they do not present any new difficulty.

One solves again perturbatively Eqs. (15) and (32) in the same way as we solved Eq. (13). We give in the Appendix the expansion of the velocity [Eqs. (A1) and (A2)] up to the fourth order and the expansions of the diffusion tensor [Eq. (A3)] and of the conductivity up to the second order [Eq. (A4)]. We are now going to illustrate these expansions by two examples: the conductivity in the symmetric case and the velocity in the nonsymmetric case.

\section{APPLICATIONS}

The first thing we did was to verify that in the weak disorder limit these expansions agree with the exact expressions of the velocity and of the diffusion constant in one dimension with nearest-neighbor hopping rates. ${ }^{21}$ However, it is clear that there are a lot of situations where the expansions can give interesting information. For example, in one dimension, it is a way to study cases which are not exactly solvable: the case of hopping rates to an arbitrary number of neighbors, strip geometries, etc. In all dimensions it can also be used in several situations and it would be tedious to discuss all of them. Therefore, we have chosen to give here only two examples: the symmetric case and the velocity in the nonsymmetric case.

\section{A. The symmetric case}

In the symmetric case one considers that the $W_{x, x^{\prime}}$ verify for any choice of $x$ and $x^{\prime}$

$$
W_{x, x^{\prime}}=W_{x^{\prime}, x}
$$

If we look at Eqs. (3) and (4), one finds that (46) implies that

$$
\begin{aligned}
& a\left(x-x^{\prime}\right)=a\left(x^{\prime}-x\right) \\
& \delta W_{x, x^{\prime}}=\delta W_{x^{\prime}, x} \\
& c_{n p}\left(x-x^{\prime}\right)=c_{n 0}\left(x-x^{\prime}\right)=c_{n n}\left(x-x^{\prime}\right) .
\end{aligned}
$$

When one uses these relations in the expressions (A1)-(A4) of the Appendix, one finds that the velocity vanishes as it should in the symmetric case and that the conductivity tensor is given by

$$
\sigma_{\mu \nu}=\sum_{z} z_{\mu} z_{\nu} a(z)+2 \sum_{z} z_{\mu} z_{\nu} c_{20}(z) \frac{1}{(2 \pi)^{d}} \int \cdots \int d^{d} q \frac{e^{i q z}-1}{i \omega+\sum_{y} a(y)\left(1-e^{i q y}\right)}
$$

It is easy to check that the diffusion tensor $D_{\mu \nu}$ is related to the conductivity $\sigma_{\mu \nu}$ in the limit $\omega \rightarrow 0$

$$
2 D_{\mu \nu}=\lim _{\omega \rightarrow 0} \sigma_{\mu \nu}
$$

It is interesting to compare the content of (50) with the effective-medium approximation. ${ }^{4,5,27}$ To do so we take the formulas (17) and (18) of Ref. 4 (see also Ref. 5). For a hypercubic lattice in dimension $d$, the conductivity $\sigma_{\text {em }}$ in the effective-medium approximation is given by

$$
\sigma_{\mathrm{em}}=d W_{m}
$$

where $W_{m}$ is a function of $\omega$ given by the following equation:

$$
\left\langle\frac{W_{m}-W_{x, x^{\prime}}}{W_{x, x^{\prime}}\left[1-i \omega G_{0}(i \omega)\right]+\left[(d-1)+i \omega G_{0}(i \omega)\right] W_{m}}\right\rangle=0
$$

where $W_{x, x^{\prime}}$ is a hopping rate, the average must be taken over the distribution of $W_{x, x^{\prime}}$, and $G_{0}(i \omega)$ is defined by

$$
G_{0}(i \omega)=\frac{1}{(2 \pi)^{d}} \int \cdots \int d^{d} q \frac{1}{i \omega+2 d-2\left(\cos q_{1}+\cdots+\cos q_{d}\right)} .
$$


When one calculates $W_{m}$ in cases where the distribution of $W_{x x^{\prime}}$ is very narrow, one finds that the effectivemedium prediction reduces to (50), except a factor $d / 2$ which comes just from the definition of the conductivity. Therefore, up to first order in this weak disorder expansion, the effective-medium approximation is exact.

\section{B. The nonsymmetric case}

Let us now investigate the nonsymmetric case, i.e., the case where the matrix $W_{x, x^{\prime}}$ is not symmetric. The first thing one can notice in the expressions (A3) and (A4) of the diffusion tensor and of the conductivity is that $D_{\mu \nu}$ is symmetric whereas $\sigma_{\mu \nu}$ is not symmetric:

$$
\begin{aligned}
& D_{\mu \nu}=D_{\nu \mu}, \\
& \sigma_{\mu \nu} \neq \sigma_{v \mu} .
\end{aligned}
$$

Therefore, the Einstein relation (51) is no longer valid here. This is not very surprising since, in general, there is just a dynamical steady state but no equilibrium which corresponds to the nonsymmetric case.

When one looks at the expressions which give the velocity, the diffusion tensor, and the conductivity, one sees that all the terms of the weak disorder expansion are sums of integrals which contain in the denominator differences such as $A(0)-A(q)$,

$$
A(0)-A(q)=\sum_{\overrightarrow{\mathrm{z}}} a(z)\left(1-e^{i q z}\right) .
$$

These denominators can vanish only at $q=0$.

Around $q=0, A(0)-A(q)$ has the following expansion:

$$
A(0)-A(q)=-i \overrightarrow{\mathrm{V}}_{0} \cdot \overrightarrow{\mathrm{q}}+O\left(q^{2}\right),
$$

where $\overrightarrow{\mathrm{V}}_{0}$ is the velocity in the pure system

$$
\overrightarrow{\mathrm{V}}_{0}=\sum_{z} \overrightarrow{\mathrm{z}} a(z)
$$

It is then clear that when $V_{0}$ goes to zero, the expansion may become singular.

An analysis of the expansion of $V_{\mu}$ [see (A1) and (A2)] in the small- $V_{0}$ limit leads to a behavior which depends strongly on the dimensionality. Let us assume for simplicity the following isotropy properties:

$$
\begin{aligned}
& \sum_{z} z_{\mu} z_{v} a(z)=2 D_{0} \delta_{\mu v}, \\
& \sum_{z} z_{\mu} z_{v}\left[c_{20}(z)-c_{21}(z)\right]=c \delta_{\mu v}
\end{aligned}
$$

( $D_{0}$ is the diffusion constant of the pure case, and $c$ is a natural measure of disorder).

Using this notation, we get the following behaviors (in the $V_{0} \rightarrow 0$ limit): for $d>2$

$$
V_{\mu}=V_{\mu}^{0}(1+\text { const } c+\cdots) \text {, }
$$

and for $d=2$

$$
V_{\mu}=V_{\mu}^{0}\left(1-\Gamma+3 \Gamma^{2}+\cdots\right)
$$

with

$$
\Gamma=\frac{c}{4 \pi D_{0}} \ln \left[\frac{D_{0}^{2}}{V_{0}^{2}}\right] .
$$

For $1<d<2$

$$
V_{\mu}=V_{\mu}^{0}\left[1-\text { const } c\left|V_{0}\right|^{-(2-d)}+\cdots\right] \text {, }
$$

and for $d=1$

$$
V=V_{0}-\operatorname{sgn}\left(V_{0}\right)\left(\frac{c}{2 D_{0}}+\cdots\right) .
$$

For $d \leq 2$, we think that each new term in the expansion is more singular in the limit $V_{0} \rightarrow 0$. It is easy to verify that this behavior in one dimension agrees with the exact expression $^{21}$ in the limit $V_{0} \rightarrow 0$.

The first important result which comes out from these behaviors near $\vec{V}_{0}=0$ is that the upper critical dimension is 2 ,

$$
d_{c}=2 \text {. }
$$

For $d>2$ velocity $V$ vanishes linearly with $V_{0}$ [Eq. (58)] and the only effect of a weak disorder is to renormalize the slope. In the contrary case, for $d \leq 2$, the expansion near $V_{0}$ is singular [Eqs. (59) and (60)], and one needs to resum the singular expansion. The best way to do it is to use the renormalization-group techniques.

The method is described elsewhere. ${ }^{25}$ One of the main results of field-theory approach is that the velocity vanishes in $d=2$ in the following way:

$$
V \sim \frac{V_{0}}{c \ln \left(\text { const } / V_{0}^{2}\right)} .
$$

This behavior shows that in $d=2$ there is just a critical point of $\vec{V}$ at $\vec{V}_{0}=0$, in agreement with what Paladin and Vulpiani ${ }^{26}$ claim. This is in contrast with $d=1$, where the velocity $V$ vanishes ${ }^{19}$ in a whole phase.

\section{CONCLUSION}

In this paper we have presented a way of calculating the velocity, the diffusion tensor, and the conductivity on a random lattice. To do so, one needs to solve Eqs. (13), (15), and (32). This could be done numerically for small samples. Here we have followed this approach to obtain weak disorder expansions for hypercubic lattices of arbitrary dimension. In principle these expansions can be carried out to any order, although the calculations become longer and longer. We have looked at the consequences of our expansion in the symmetric case: We find that the effective-medium theory gives the first term of the expansion correctly. Therefore, one needs more terms in the expansion to see where the effective-medium theory breaks down.

For the nonsymmetric case, we have seen that our weak 
disorder expansion shows that $d_{c}=2$ is the upper critical dimension. The field theoretical approach leads to the behavior (63) for the velocity $V$ in the limit $V_{0} \rightarrow 0$ in $d=2$. This behavior will be justified in a planned forthcoming work $^{25}$ by using renormalization-group techniques. We expect the $\log ^{-1}$ behavior (63) to be observable in a region which vanishes for small disorder $\left(\left|\log V_{0}\right| \gg 1 / c\right)$. It would be interesting to know if this prediction (63) could be seen in numerical simulations or in experimental situations.

\section{ACKNOWLEDGMENTS}

We would like to thank R. Orbach and I. Webman for several discussions which motivated this work, and L. Peliti for interesting remarks.

\section{APPENDIX}

In this appendix we give the weak disorder expansions of the velocity, the diffusion tensor, and the conductivity tensor. We have carried out the expansion of the velocity up to the fourth power in the $\delta W_{x, x^{\prime}}$. The result is

$$
V_{\mu}=\sum_{z} z_{\mu} B(z)
$$

where $B(z)$ is given by

$$
\begin{aligned}
B(z)= & a(z)+\left[c_{20}(z)-c_{21}(z)\right] I(z)+\left[c_{30}(z)-c_{31}(z)\right] I^{2}(z)+2 c_{31}(z) I(z) I(-z) \\
& +\left\{c_{40}(z)-c_{41}(z)-3 c_{20}(z)\left[c_{20}(z)-c_{21}(z)\right]\right\} I^{3}(z) \\
& +3\left\{c_{41}(z)-c_{42}(z)+c_{21}(z)\left[c_{20}(z)-c_{21}(z)\right]\right\} I^{2}(z) I(-z) \\
& +c_{20}(z) \sum_{y}\left\{c_{20}(y)\left[I_{2}(z, y)+I_{3}(z, y) I(z)\right]+c_{21}(y)\left[I_{3}(z,-y) I(y)-I_{2}(z, y)\right]\right\} \\
& -c_{21}(z) \sum_{y}\left\{c_{20}(y)\left[I_{4}(z, y)+I_{3}(z, y) I(y)\right]+c_{21}(y)\left[I_{3}(z,-y) I(y)-I_{4}(z, y)\right]\right\},
\end{aligned}
$$

where the functions $I, I_{2}, I_{3}$, and $I_{4}$ are defined by

$$
I(z)=\frac{1}{(2 \pi)^{d}} \int_{0}^{2 \pi} \cdots \int_{0}^{2 \pi} d^{d} q \frac{e^{i q z}-1}{A(0)-A(q)}
$$

[one should notice that $I(z)$ is just a way of rewriting $G(z, 0)$ since $I(z)=G(z, 0)$ ],

$$
\begin{aligned}
& I_{2}(z, y)=\frac{1}{(2 \pi)^{2 d}} \int \cdots \int d^{d} p d^{d} q \frac{\left(e^{i(p+q) z}-1\right)\left(e^{i p y}-1\right)\left(e^{i q y}-1\right)}{[A(0)-A(p+q)][A(0)-A(p)][A(0)-A(q)]}, \\
& I_{3}(z, y)=\frac{1}{(2 \pi)^{d}} \int \cdots \int d^{d} q \frac{\left(e^{i q z}-1\right)\left(e^{i q y}-1\right)}{[A(0)-A(q)]^{2}}, \\
& I_{4}(z, y)=\frac{1}{(2 \pi)^{2 d}} \int \cdots \int d^{d} p d^{d} q \frac{\left(e^{i p y}-1\right)\left(e^{i q y}-1\right)\left(e^{i p z}-e^{-i q z}\right)}{[A(0)-A(p)][(A(0)-A(q)][(A(0)-A(p+q)]},
\end{aligned}
$$

and where $A(p)$ is defined by

$$
A(p)=\sum_{z} a(z) e^{i p z}
$$

We have also obtained the weak disorder expansions of the diffusion tensor and the conductivity tensor,

$$
D_{\mu \nu}=\frac{1}{2} \sum_{z} z_{\mu} z_{v} a(z)+\frac{1}{2} \sum_{z} z_{\mu} z_{\nu}\left[c_{20}(z)+c_{21}(z)\right] I(z)+\frac{1}{2} \sum_{z, y}\left[z_{\mu} y_{\nu} a(y)+z_{v} y_{\mu} a(y)\right]\left[c_{20}(z)-c_{21}(z)\right] J(z)
$$

where $I$ has been defined and where $J$ is defined by

$$
\begin{aligned}
J(z)= & \frac{1}{(2 \pi)^{d}} \int \cdots \int d^{d} q \frac{e^{i q z}-1}{[A(0)-A(q)]^{2}} \\
\sigma_{\mu \nu}= & \sum_{z} z_{\mu} z_{v} a(z)+\sum_{z, y} z_{\mu} z_{v}\left[c_{20}(z)+c_{21}(z)\right] H(z, \omega)+\sum_{z} z_{\mu} y_{v} a(y)\left[c_{20}(z)-c_{21}(z)\right] H_{2}(z, y, \omega) \\
& +\sum_{z} z_{\mu} z_{v}\left[c_{20}(z)-c_{21}(z)\right] I(z)
\end{aligned}
$$


where

$$
\begin{aligned}
& H(z, \omega)=\frac{1}{(2 \pi)^{d}} \int \cdots \int d^{d} q \frac{e^{i q z}-1}{i \omega+A(0)-A(q)} \\
& H_{2}(z, y, \omega)=\frac{1}{(2 \pi)^{d}} \int \cdots \int d^{d} q \frac{\left(e^{i q z}-1\right)\left(e^{i q y}-1\right)}{[i \omega+A(0)-A(q)][A(0)-A(q)]} .
\end{aligned}
$$

${ }^{1}$ S. Alexander, J. Bernasconi, W. R. Schneider, and R. Orbach, Rev. Mod. Phys. 53, 175 (1981).

${ }^{2}$ S. Alexander, J. Bernasconi, W. R. Schneider, R. Biller, W. G. Clark, G. Grüner, R. Orbach, and A. Zettl, Phys. Rev. B 24, 7474 (1981)

${ }^{3}$ R. Zwanzig, J. Stat. Phys. 28, 127 (1982).

${ }^{4}$ I. Webman, Phys. Rev. Lett. 47, 1496 (1981).

${ }^{5}$ I. Webman and J. Klafter, Phys. Rev. B 26, 5950 (1982).

6. W. Haus, K. W. Kehr, and K. Kitahara, Phys. Rev. B 25, 4918 (1982).

7J. Machta, Phys. Rev. B 24, 5260 (1981).

${ }^{8}$ M. J. Stephen and R. Kariotis, Phys. Rev. B 26, 2917 (1982).

${ }^{9}$ P. G. de Gennes, La Recherche 7, 919 (1976).

${ }^{10}$ C. D. Mitescu, H. Ottavi, and J. Roussenq, Electrical Transport and Optical Properties of Inhomogeneous Media (Ohio State University, (1977), Proceedings of the First Conference on the Electrical Transport and Optical Properties of Inhomogeneous Media, edited by J. C. Garland and D. B. Tanner (AIP, New York, 1978); C. D. Mitescu and J. Roussenq, Ann. Isr. Phys. Soc. Israel 5, 101 (1983).

11 J. P. Straley, J. Phys. C $\underline{13}$, 2991 (1980).

${ }^{12}$ D. Ben-Avraham and S. Havlin, J. Phys. A 15, L691 (1982).

${ }^{13}$ M. Sahimi, B. D. Hughes, L. E. Scriven, and H. T. Davis, J. Chem. Phys. 78, 6849 (1983).
${ }^{14}$ R. Rammal and G. Toulouse, J. Phys. Lett. (Paris) 44, L13 (1983).

${ }^{15}$ S. Alexander and R. Orbach, J. Phys. Lett. (Paris) $\underline{43}$, L625 (1982).

${ }^{16}$ H. Kesten, M. V. Kozlov, and F. Spitzer, Compositio Math. 30, 145 (1975).

${ }^{17}$ F. Solomon, Ann. Prob. $\underline{3}, 1$ (1975).

${ }^{18}$ Ia. G. Sinai, in Proceedings of the Berlin Conference on Mathematical Problems in Theoretical Physics, Vol. 153 of Lectures Notes in Physics, edited by R. Schrader, R. Seiler, and D. A. Unlenbrock (Springer, Berlin, 1982), p. 12.

${ }^{19}$ B. Derrida and Y. Pomeau, Phys. Rev. Lett. $\underline{48}, 627$ (1982).

20J. Bernasconi and W. R. Schneider, J. Phys. A15, L729 (1982).

${ }^{21}$ B. Derrida, J. Stat. Phys. 31, 433 (1983).

${ }^{22}$ B. Derrida and R. Orbach; Phys. Rev. B 27, 4694 (1983).

${ }^{23}$ M. Barma and D. Dhar, J. Phys. C 16,1451 (1983).

${ }^{24}$ E. Marinari, G. Parisi, D. Ruelle and P. Windey; Comm. Math. Phys. (in press); Phys. Rev. Lett. 으, 1223 (1983).

25J. M. Luck, Centre d'Etudes Nucléaires de Saday Report No. SPh.T/83/30 (unpublished); Nucl. Phys. B 225 [FS9], 169 (1983).

${ }^{26}$ G. Paladin and A. Vulpiani, J. Phys. Lett. (Paris) 44 , L443 (1983). 